\title{
La génétique moléculaire de la rétinite pigmentaire : après la rhodopsine, la périphérine
}

La génétique moléculaire des affections rétiniennes, et surtout des rétinites pigmentaires, progresse à pas de géant. A peine avions-nous décrit les mutations nombreuses de la rhodopsine $\left(\mathrm{m} / \mathrm{s} n^{\circ} 1\right.$, vol. 8, p. 81) qu'un nouveau gène candidat entre en lice. Ce gène a été identifié comme étant celui de la périphérine, une glycoprotéine spécifique des cellules photoréceptrices. Son gène a été d'abord localisé chez la souris sur le chromosome 17 [1] puis chez l'homme sur le bras court du chromosome 6 au voisinage du centromère [2]. Dans les deux espèces - et dans d'autres étudiées depuis, - la protéine compte 346 acides aminés, sans peptide signal, et quatre domaines hydrophobes probablement transmembranaires. La périphérine est le produit normal d'un gène, qui, muté chez la souris, provoque une dégénćrescence lente de la rétine ( $r d s$, retinal degeneration slow). La lésion moléculaire de la souris est une insertion d'environ $10 \mathrm{~kb}$ au sein d'un exon. Deux équipes, les plus actives dans ce domaine, ont recherché, chez des malades atteints de la forme autosomique dominante de la rétinite pigmentaire, si la périphérine ne pouvait être en cause.

Farrar et al. (Dublin, Irlande) ont étudié 60. sujets non apparentés (en éliminant les porteurs d'une lésion de la rhodopsine), ainsi qu'une famille irlandaise comportant 72 membres [3, 4]. L'ensemble de leurs investigations a permis de localiser le locus responsable au bras court du chromosome 6 et, plus précisément, sur la périphérine.

\footnotetext{
* Le SSCP ou, en francais, le "polymorphisme de conformation des brins séparés d'ADN", est fondé sur la migration en gel non dénaturant de polyacrylamide d'un fragment d'ADN dont les brins ont été préalablement séparés. Dans ces conditions, la migration électrophorétique des brins dépend de leur conformation, elle-même déterminée par leur séquence. De ce fait, tout changement de séquence peut entraîner une modification de mobilité.

$\mathrm{m} / \mathrm{s} n^{\circ} 2$, vol. 8, février 92
}

L'amplification de l'ADN et la méthode du $\mathrm{SSCP}^{*}$ (single strand conformation polymorphysm) ont montré chez un malade, une délétion portant sur une des deux cystéines des codons 118 et 119 , au niveau de $3^{\mathrm{e}}$ domaine transmembranaire de la périphérine. Dans la famille de ce malade la délétion coségrège avec l'atteinte clinique.

Le groupe de Dryja, qui compte déjà à son actif la découverte de la plupart des mutations de la rhodopsine (Boston, MA, et Dallas, TX, USA) a suivi [5] une voie un peu différente. Il est parti d'un $\mathrm{ADNc}$ murin pour isoler celui de l'homme. Il a examiné 106 malades non apparentés, par les mêmes méthodes, et a découvert trois mutants, qui tous mettaient en cause une proline : un changement d'une leucine en proline en position 185 (retrouvé chez deux malades non apparentés), d'une proline en leucine en 216 , et une délétion d'une proline en 219. De plus, dans trois des familles, tous les membres atteints, et eux seuls, portaient la mutation. Toutes ces mutations se localisaient dans une zone de la molécule très conservée, probablement extracytoplasmique.

L'existence d'une maladie humaine avec une contrepartie animale pose un problème génétique intriguant. La rétinite pigmentaire liée au chromosome 6 a une transmission dominante et n'est connue que chez des hétérozygotes. Celle de la souris est semidominante. Homo et hétérozygotes montrent un développement anormal des photorécepteurs avec dégénérescence progressive, mais celle-ci est beaucoup plus rapide chez les homozygotes. Cette observation conduit à rechercher l'existence d'homozygotes dans la maladie humaine, mais aussi des anomalies récessives de ce gène. Enfin il se pour- rait que le nombre des mutants se révèle finalement plus élevé, car il ne semble pas que les amplifications réalisées aient couvert la totalité de l'ADNc. Par ailleurs, le gène de la périphérine est très proche du locus HLA auquel il est étroitement lié. Travis et al. [2] font remarquer que le gène SCA de l'ataxie cérébelleuse dominante est également très proche. Ils suggèrent l'étude d'éventuels malades atteints à la fois de SCA et de rétinite pigmentaire, à la recherche d'une délétion interstitielle englobant les deux loci.

La périphérine constitue donc, après la rhodopsine, la deuxième protéine des photorécepteurs dont une lésion entraîne une rétinite pigmentaire. De plus, d'autres modèles de la maladie sont déjà à l'étude : tout d'abord, une forme dominante de la rétinite a vu son gène assigné au bras long du chromosome 3 , comme celui de la rhodopsine, mais en une position différente, puisque aucune liaison entre les deux n'a été trouvée [6]. Surtout, une équipe de Los Angeles (CA, USA), et Houston (TX, USA), a pu montrer qu'une famille, initialement originaire du Kentucky, et dont on connaît 600 membres, présente une forme de rétinite pigmentaire dont le gène peut être localisé dans la région péricentrique du chromosome 8 , au voisinage de ceux de ANK1 (ankyrine), et de PLAT (activateur tissulaire du plasminogène [7]. Ce gène n'a pas été encore isolé.

Ajoutons qu'un groupe international de 23 auteurs [8] s'est efforcé de placer au long du chromosome $\mathrm{X}$ les deux (ou peut-être trois) loci responsables de formes récessives liées au sexe - seules les formes récessives autosomiques n'ont pu être encore localisées. On peut donc classer de la façon suivante 
les loci identifiés actuellement, selon une nomenclature qui semble admise, mais qui mériterait révision quand les données connues seront plus complètes :

$\begin{array}{lll}\text { RP1 }: 8 p & \text { RP2 }: \text { Xp 12 } & \text { RP3 }: \text { Xp21 } \\ \text { RP4 }: 3 q \text { (rhodopsine) } & \text { RP5 : 3q (non rhodopsine) } & \text { RP6 }: 6 p\end{array}$

J.C. D.

1. Travis $\mathrm{GH}$, Brennan $\mathrm{MB}$, Danielson $\mathrm{PE}$, Kozak CA, Sutcliffe JG. Identification of a photoreceptor-specific mRNA encoded by the gene responsible for retinal degeneration slow (rds). Nature 1989 ; 338 : 70-3.

2. Travis GH, Christerson L, Danielson PE, et al. The human retinal degeneration slow (RDS) gene : chromosome assignment and structure of the mRNA. Genomics $1991 ; 10: 733-9$.

3. Farrar GJ, Kenna P, Jordan SA, el al. A three-base-pair deletion in the peripherin-RDS gene in one form of retinitis pigmentosa. Nature $1991 ; 354$ : 478-80.

4. Farrar GJ, Jordan SA, Kenna P, et al. Autosomal dominant retinitis pigmentosa : localization of a disease gene (RP6) to the short arm of chro mosome 6. Genomics 1991; 11: 870-4.
5. Kajiwara K, Hahn LB, Mukai S, Travis $\mathrm{GH}$, Berson EL, Dryja TP. Mutations in the human retinal degeneration gene in autosomal dominant retinitis pigmentosa. Nature 1991; 354 : 480-3.

6. Olsson JE, Sammans C, Jimenez J, et al. Gene of type II autosomal dominant retinitis pigmentosa maps on the long arm of chromosome 3 Am J Med Genet 1990 ; 35 : 595-9.

7. Blanton SH, Heckenlively JR, Cottingham AW, el al. Linkage mapping of autosomal dominant retinitis pigmentosa (RP1) to the pericentric region of chromosome 8 . Genomics 1991; 11: 857-69.

8. Ott J, Bhattacharya S, Chen JD, et al. Localizing multiple $\mathrm{X}$ chromosome-linked retinitis pigmentosa loci using multilocus homogeneity tests. Proc Nall Acad Sci USA 1990 ; 87 : 701-04. 